\title{
Nanoscale enabled Future Healthcare using Terahertz
}

\author{
Qammer H. Abbasi \\ University of Glasgow \\ 1 University Ave, Glasgow G12 8QQ, United Kingdom \\ Qammer.Abbasi@glasgow.ac.uk
}

\begin{abstract}
Advancement in nanotechnology has made it possible to manufacture sensors, circuits and devices measuring only nanometers in size. This development is creating an extraordinary opportunity to observe, interact, and optimize physical systems from the very bottom. Wireless communication and networking at nanoscale, however, faces new challenges not encountered in conventional sensor networks. For example, nanoscale antenna call for wireless communication in the Terahertz band, which encounters new path loss and noise phenomena posing significant challenges for many target applications of such networking. Nanoscale computing and communication is a new and rapidly growing field of research promoting collaboration between wireless networking, nanotechnology, and other fundamental disciplines. However, the research is in its early stages to realize communication and networking at the nanoscale. The talk will present the opportunities, challenges, and recent advancements of this new and growing inter-disciplinary field explicitly in healthcare and agricultural technology domains.
\end{abstract}

